\title{
Video Article \\ Longitudinal Intravital Imaging of Brain Tumor Cell Behavior in Response to an Invasive Surgical Biopsy
}

\author{
Maria Alieva ${ }^{1}$, Anne C. Rios $^{1}$ \\ ${ }^{1}$ Cancer Genomics Netherlands, Prinses Máxima Center for Pediatric Oncology \\ Correspondence to: Maria Alieva at M.Alieva@prinsesmaximacentrum.nl \\ URL: https://www.jove.com/video/59278 \\ DOI: doi:10.3791/59278
}

Keywords: Cancer Research, Issue 147, Intravital imaging, glioma, biopsy, migration, proliferation, cranial imaging window

Date Published: 5/3/2019

Citation: Alieva, M., Rios, A.C. Longitudinal Intravital Imaging of Brain Tumor Cell Behavior in Response to an Invasive Surgical Biopsy. J. Vis. Exp. (147), e59278, doi:10.3791/59278 (2019).

\section{Abstract}

Biopsies are standard of care for cancer treatment and are clinically beneficial as they allow solid tumor diagnosis, prognosis, and personalized treatment determination. However, perturbation of the tumor architecture by biopsy and other invasive procedures has been associated with undesired effects on tumor progression, which need to be studied in depth to further improve the clinical benefit of these procedures. Conventional static approaches, which only provide a snapshot of the tumor, are limited in their ability to reveal the impact of biopsy on tumor cell behavior such as migration, a process closely related to tumor malignancy. In particular, tumor cell migration is the key in highly aggressive brain tumors, where local tumor dissemination makes total tumor resection virtually impossible. The development of multiphoton imaging and chronic imaging windows allows scientists to study this dynamic process in living animals over time. Here, we describe a method for the high-resolution longitudinal imaging of brain tumor cells before and after a biopsy in the same living animal. This approach makes it possible to study the impact of this procedure on tumor cell behavior (migration, invasion, and proliferation). Furthermore, we discuss the advantages and limitations of this technique, as well as the ability of this methodology to study changes in the cancer cell behavior for other surgical interventions, including tumor resection or the implantation of chemotherapy wafers.

\section{Video Link}

The video component of this article can be found at https://www.jove.com/video/59278/

\section{Introduction}

Standard of care for most solid tumors includes tissue biopsy for diagnosis, prognosis, and personalized treatment determination ${ }^{1,2}$. Overall, these procedures give clinical benefit, but recent evidence indicates that biopsy and other more invasive procedures, such as tumor resection, can also negatively influence tumor progression ${ }^{3,4,5,6}$. While these procedures remain indispensable in-patient care and their benefits overcome their negative effects, it is necessary to fully understand the mechanisms behind these negative effects in order to maximize the patients' safety and the positive influences of these procedures and make them even more clinically beneficial.

Biopsy-mediated undesired effects on tumor progression are triggered by systemic alterations and changes in the tumor microenvironment in response to tissue disruption ${ }^{4,5}$. Thus, it is necessary to study this process in live animals. However, the subtle consequences of these minimally invasive procedures can often be disguised by large variations between individuals. Conventional methods based on immunohistochemistry or transcriptional expression analysis may overlook these effects or require large numbers of animals to identify them. Moreover, these static approaches lack the ability to identify changes in tumor cell behavior such as migration and invasion, dynamic processes that correlate with tumor malignancy. These tumor cell features are of particular importance for highly aggressive brain tumors, such as glioblastoma multiforme (GBM), where the local spreading of tumor cells limits surgical resection and decreases patient survival ${ }^{7}$. To fully understand how biopsies affect the behavior of GBM cells, a longitudinal approach that allows visualization of these cells in the physiological context of living organisms is needed.

The recent development of high-resolution intravital imaging in combination with surgically implanted chronic imaging windows allows scientists to study the dynamic behavior of tumor cells in living mice over multiple days ${ }^{8,9}$. Using this powerful approach, we can study how tumor cells' proliferative, migratory, and infiltrative behavior changes over several days in response to a biopsy in the same mouse. Compared to other techniques that allow multi-day monitoring of tumors in live mice, such as magnetic resonance imaging (MRI) ${ }^{10}$, positron emission tomography/ computed tomography $(\mathrm{PET} / \mathrm{CT})^{11}$, or bioluminescent imaging ${ }^{12}$, this approach uniquely offers the possibility of studying the tumor cell behavior at single cell level and unraveling subtle changes occurring within the tumor.

Here, we describe a detailed method to perform biopsy-like injury and pre- and postbiopsy longitudinal intravital imaging in the brain of tumorbearing mice. This method can potentially be applied to study other surgical interventions, such as partial tumor resection or the implantation of chemotherapy wafers. 


\section{Protocol}

All experiments were carried out in accordance with the guidelines of the Animal Welfare Committee of the Royal Netherlands Academy of Arts and Sciences, the Netherlands. The experimental protocols used in this manuscript were approved by the Centrale Commissie Dierproeven (CCD) and the Instantie voor Dierenwelzijn (IvD).

\section{Tumor cell implantation and cranial imaging window preparation}

\section{Surgical preparation}

1. Use adult mice (>6 weeks old) of any strain or gender. Sedate a mouse by injecting (fluanisone [neuroleptic] + fentanyl [opioid]) ( 0.4 $\mathrm{mL} / \mathrm{kg})+$ benzodiazepine sedative $(2 \mathrm{mg} / \mathrm{kg})$ at a dose of 1:1:2 in sterile water. Assess the animal's sedation state by toe pinch. NOTE: In this protocol, both female and male C57BL/6 mice were used due to the same genetic background of the tumor cell line used in these experiments (GL261). The mouse will stay fully sedated for $1.5 \mathrm{~h}$. Alternatively, use inhalation anesthesia such as isoflurane $\left(1.5 \%-2 \%\right.$ isoflurane $/ \mathrm{O}_{2}$ mixture).

2. Mount the mouse on a stereotactic frame and secure the head using a nose clamp and two ear bars.

3. Use a heating lamp to preserve body temperature. Heating lamp should be used with caution, alternatively water recirculating heating pads can be used.

4. Apply eye ointment to protect the mouse's corneas from drying.

5. Use sharp scissors to shave the fur on the skull (dorsal area from the mouse's eyes to the base of its skull) and disinfect the exposed skin with $70 \%$ ethanol.

6. Cut the skin in a circular manner with sharp scissors and scrape away the periosteum underneath with a cotton swab. Apply a drop of lidocaine $1 \%$ + epinephrine 1:100,000 for 5 min, and remove the excess with a cotton swab.

7. Glue the edges of the skin to the skull with cyanoacrylate glue.

8. Place the stereotactic frame under a dissection stereo microscope with $4 x$ magnification.

9. Visualize the skull through the dissection microscope and drill a circular groove of $5 \mathrm{~mm}$ in diameter over the right parietal bone. Perform this step carefully and only superficially, avoiding any pressure on the skull.

10. Apply a drop of cortex buffer $\left(125 \mathrm{mM} \mathrm{NaCl}, 5 \mathrm{mM} \mathrm{KCl}, 10 \mathrm{mM}\right.$ glucose, $10 \mathrm{mM} \mathrm{HEPES} \mathrm{buffer,} 2 \mathrm{mM} \mathrm{MgSO}$, and $2 \mathrm{mM} \mathrm{CaCl}_{2}[\mathrm{pH}$ $7.4]$ ) and lift the bone flap using thin forceps.

11. For the following steps, keep the brain surface covered with cortex buffer unless indicated otherwise.

12. Under the dissection microscope, visualize the brain surface and remove the dura mater using curved, tapered, very fine point tweezers. If bleeding occurs at this stage, use an absorbable gelatin sponge to stop it.

\section{Tumor cell injection}

1. Resuspend the desired amount of fluorescent tumor cells in $\sim 3 \mu \mathrm{L}$ of PBS (e.g., for the experiments shown in this protocol, $1 \times 10^{5}$ GL261 cells were injected).

NOTE: While any fluorescent marker can be used, a nuclear marker is strongly advised, to track individual tumor cells during analysis. Additionally, a histone-linked fluorescent marker, such as $\mathrm{H} 2 \mathrm{~B}$, can be used to visualize chromosome condensation and, thus, to monitor cell division. The use of a photo-switchable marker, such as Dendra2, allows the photo-marking and tracking of tumor cells over several days ${ }^{4,13}$.

2. Load the tumor cells in a $10 \mu \mathrm{L}$ gas-tight syringe with a point style 2 needle and fix it on the stereotaxic manipulator arm.

3. Remove the cortex buffer. Place the tip of the syringe in the middle of the craniotomy and insert it at a depth of $0.5 \mathrm{~mm}$ from the surface of the skull. Optionally, create a small space in the brain to accommodate the tumor cell suspension. For this, insert the syringe up to a depth of $1 \mathrm{~mm}$ and then retrieve it up to $0.5 \mathrm{~mm}$ before the injection.

4. Apply a drop of cortex buffer.

5. Slowly inject the cell suspension using a microsyringe pump injector (250-400 $\mathrm{nL} / \mathrm{min})$. Remove the syringe and use an absorbable gelatin sponge to stop any bleeding, if necessary.

\section{Cranial imaging window preparation}

1. Remove the cortex buffer and place a drop of silicone oil on the craniotomy site to avoid air bubbles under the window.

2. Seal the exposed brain with a $6 \mathrm{~mm}$ coverslip. Apply cyanoacrylate glue between the coverslip and the skull. Gently press the coverslip against the skull with the help of fine tweezers to ensure minimal distance between the brain and the coverslip.

3. Apply dental acrylic cement on the skull's surface, covering the edge of the coverslip. Place a thin stainless-steel ring (1.5 mm in outer diameter, $1 \mathrm{~mm}$ in inner diameter) around the craniotomy and allow the dental cement to dry. Optionally, add some glue on the border to secure the ring on the top of the head.

NOTE: This head fixation system is optimal for imaging on an inverted microscope: small magnets embedded in the imaging box facilitate the cranial imaging window (CIW) fixation. In the experiments shown in this protocol, an inverted microscope was used. For upright microscopes, fixation can be achieved by means of a bar or a ring with grooves that can be attached to the microscope with the help of screws or a plate that fits the ring.

4. For pain management, inject a single dose of $100 \mu \mathrm{g} / \mathrm{kg}$ buprenorphine subcutaneously and allow the animal to recover on a heating pad.

5. Place the mouse in an individual cage with shredded paper for enrichment. Closely monitor the mouse daily for the first few days and $2 x$ per week, thereafter, for normal behavior, reactivity, and appearance. 


\section{Intravital imaging}

NOTE: The time interval between the tumor cell injection and the first intravital imaging session is dependent on the type of tumor cell line used. For the experiments shown in this protocol, $1 \times 10^{5} \mathrm{GL} 261$ cells were injected and imaged 10 days later.

1. Imaging preparation

1. Sedate the mouse using isoflurane inhalation anesthesia through a face mask $\left(1.5 \%-2 \%\right.$ isoflurane $/ \mathrm{O}_{2}$ mixture).

2. Inject the mouse subcutaneously with $100 \mu \mathrm{L}$ of saline buffer to prevent dehydration.

NOTE: For long-term imaging, the mouse can be hydrated through a subcutaneous infusion pump.

3. Place the mouse face-up in an imaging box. Use a metal plate with a $1 \mathrm{~mm}$-diameter hole and small magnets embedded around the aperture to provide fixation of the CIW to the imaging box. Introduce isoflurane through a facemask and ventilate by an outlet on the other side of the box $\left(0.8 \%-1.5 \%\right.$ isoflurane $/ \mathrm{O}_{2}$ mixture $)$. Optionally, use tape to fix the body of the mouse.

NOTE: Fluorescently labeled dextran for blood vessel visualization or other dyes may be injected intravenously at this point.

4. Optionally, use a pulse oximeter and a heating probe to monitor the mouse's vitals.

NOTE: In this experiment, it was not necessary to use a pulse oximeter and a heating probe since the mouse was stable throughout the imaging time period $(2-3 \mathrm{~h})$. However, for longer imaging times, more thorough monitoring may be needed.

5. Set the 25x (e.g., HCX IRAPO NA0.95 WD $2.5 \mathrm{~mm}$ ) water objective to the lowest $z$-position and add a large drop of water. NOTE: The use of a water immersion micro dispenser is highly advised for long term experiments since it allows scientists to add water during the experiment. Alternatively, a dry objective can be used.

6. Transfer the imaging box onto the microscope equipped with a dark climate chamber kept at $37^{\circ} \mathrm{C}$. Bring the objective to the CIW coverslip until the water drop touches it.

7. Using the epifluorescence mode, observe the tumor through the eyepiece and bring the cells into focus.

\section{Time-lapse image acquisition}

1. Select several positions of interest to image, and record their coordinates in the software. Ensure that the selected positions are representative positions from different sites of the tumor (each tumor can be different, but to ensure consistency, select the same amount of positions that are central to the tumor core and to the edges across all mice).

NOTE: A tile scan of a part of the tumor or of the whole visible tumor may be performed; however, if the tumor is large, this method will increase the time-lapse between images. In addition, if the tumor cells move fast, it can be challenging to track the same cells over time.

2. Switch to multiphoton mode and tune the laser to the correct wavelength. Note that, to avoid photodamage, higher wavelengths are desired.

NOTE: For Dendra2 imaging, a $960 \mathrm{~nm}$ wavelength was used. With the objective used in these experiments, a zoom of 1.3 was sufficient to get a good resolution of the tumor nuclei and scan a representative area of the tumor.

3. Go to the live mode and define a $z$-stack for each position in order to acquire the maximal volume of tumor cells without compromising the tumor cell resolution. Define the step size between the images as $3 \mu \mathrm{m}$.

4. Use the bidirectional mode to increase the scanning speed. The image resolution should be at least $512 \times 512$ pixels.

5. Acquire images of the tumor volume at different positions every $20 \mathrm{~min}$ for $2 \mathrm{~h}$. Add water to the objective before each image acquisition.

NOTE: Time-lapse images can be automatically acquired by setting the right time-lapse in the software. However, the mouse can move, and the position or z-stack can shift over time, causing data loss. Therefore, it is recommended to perform the time-lapse manually and to check and adjust for $x y z$ shifts in between acquisitions.

6. At this step, optionally, photo-switch the Dendra2 fluorescent marker.

NOTE: As opposed to time-lapse imaging (which allows studying individual tumor cells properties and how they change over time), this will allow studying the area of tumor cell infiltration in the brain over several days ${ }^{4}$. A detailed explanation of this step is described by Gligorijevic et al. ${ }^{13}$.

7. After the last image is acquired, remove the mouse from the stage and allow it to recover on a heating pad. To prevent dehydration, $100 \mu \mathrm{L}$ of saline can be given subcutaneously. Place the mouse in its cage until the biopsy-like injury is performed.

\section{Biopsy-like injury and CIW replacement}

1. Create a biopsy-like injury at the tumor site 1 day after the first imaging session

NOTE: Alternatively, another procedure, like partial tumor removal, can be performed.

1. Sedate the mouse using isoflurane inhalation anesthesia as previously described in step 2.1.1. NOTE: While injectable anesthesia can be used, this procedure is shorter than the CIW implantation, and inhalation anesthesia makes it possible to precisely control and to reduce the time the mouse remains sedated.

2. Place the mouse on a stereotaxic frame and secure its head with two ear bars and a nose clamp. Check the depth of anesthesia by the lack of pedal reflexes. Use an anesthesia mask for stereotaxic surgery to keep the mouse sedated during the procedure.

3. Soak a cotton swab in acetone and spread it around the edge of the coverslip to soften the glue that holds the coverslip against the brain.

4. Slide thin point forceps under the coverslip to lift it. If the coverslip breaks at this point, use the forceps to remove the pieces of glass.

5. Apply cortex buffer to keep the brain moist.

6. Dip a $25 \mathrm{G}$ needle in the tumor to a depth of $1 \mathrm{~mm}$. Remove the needle and stop the bleeding, if necessary, by applying a gelatin sterile sponge.

NOTE: This step can be performed under a fluorescent stereomicroscope to more accurately identify the tumor area (if the tumor is too small). Additionally, a $1 \mu \mathrm{L}$ solution of fluorescent polystyrene $1 \mu \mathrm{m}$ beads can be injected during the puncture to identify the biopsied area. 
7. Seal the brain surface with silicone oil and glue a $6 \mathrm{~mm}$ coverslip on the top.

\section{Repeated imaging}

1. One day after inflicting the biopsy-like injury (and on consecutive days if needed), repeat intravital imaging as described in Step 2 above to assess how the tumor cell behavior changes over time. Select several positions of the tumor that would be representative of the whole tumor area.

NOTE: If fluorescent beads were used to identify the biopsied area, localize them to image tumor cell behavior in the biopsied regions compared to non-biopsied regions.

\section{Image analysis}

1. If the time-lapse images were acquired manually, combine them into one folder.

1. Open the time-lapse LIF file in the commercial software associated with the microscope (e.g., LasX or LAS AF). Select the tab Process $>$ Process Tools $>$ Merge. Select the first image of the time sequence and click First. Select the second image of the time sequence and click Second. In Merge Dimensions, select $\mathbf{t}$ for time. Click Apply. A new file with two timepoints will be generated. Repeat this process for all the timepoints, using the newly generated file as the first image of the sequence.

2. Correct the acquired $z$-stacks for any $x y z$ shift.

NOTE: For the experiments described in this protocol, a custom-designed Visual Basic software program was used for correction.

Alternatively, other available software can be used, such as ImageJ or Imaris.

1. Export the TIFF files from the software by right-clicking on the merged time-lapse file. Select Save RAW Data. The exported files will be exported to one folder named according to the channel, time, and z-position.

2. Open the TIFF files in the custom-designed Visual Basic software program.

3. Define the number of timepoints, $z$-stacks, and channels.

4. Assign a color to each channel by the order of appearance.

5. In the Form Show panel, for each timepoint, correct the shift in the $z$ by clicking on the up (U) or down (D) buttons.

6. In the Intravital image building panel, select the channel to be used to correct for the $x y$ shift. Select the green channel to correct, based on the signal from the tumor cells. Click Automatic for $x y$ correction.

7. Export the corrected images as a maximum projection of three consecutive z-stacks. In the panel Selection, introduce the numbers of the first (Begin) and the last (End) $Z$-slices to be exported. Select Max. Select Separate folders for Z. Click Do. For each of them, three $z$-stacks, time-lapse images will be exported to a separate folder.

3. Optionally, correct for the tissue elastic deformation.

NOTE: For tissue elastic deformation experiments, a match motion compensation software program was used to correct for rigid and elastic tissue deformation ${ }^{14}$

4. Track single cells in the complete $z$-stack in the time-lapse movie.

NOTE: Tumor cells can be tracked manually using, for instance, an ImageJ plugin (MTrackJ). While accurate, this is limited to the $x y$ plane and is very time-consuming. Alternatively, tumor cells can be tracked automatically throughout the full $z$-volume, using tumor cell segmentation (e.g., Imaris software). However, in highly dense tumors, automated tracking is less accurate and can give rise to more tracking errors.

1. Open and track each time-lapse series (for three consecutive z-stacks) separately. Drag the folder containing the time-lapse images to ImageJ.

2. Select the tab Plugins > Tracking > MtrackJ. Track each individual cell by selecting Add and clicking on each cell at each timepoint.

3. Extract the measure of the tracks by clicking on Measure and saving the file.

\section{Representative Results}

To assess the impact of biopsy on brain tumor cell behavior, we performed the procedure described in this protocol. Glioma-GL261 cells - expressing a nuclear fluorescent protein (H2B-Dendra2) were injected in the brain of C57BL/6 mice, and a chronic CIW was implanted. Time-lapse intravital imaging was performed on the same animal pre- and post-biopsy-like injury to the tumor (Figure 1A,B). The migration of individual tumor cells was determined by tracking the migration path over time in different $x y$ planes of the $z$-stack (Figure 1C) and plotted as a percentage of migratory cells pre- and postbiopsy (Figure 1F). The tumor cell proliferation rate was quantified based on H2B-tagged Dendra2 condensation upon mitosis (Figure 1D) and plotted as a percentage of dividing cells pre- and postbiopsy (Figure 1E). We compared the distribution of migration velocity before and after biopsy in the same tumor and found that the number of migratory cells (velocity $>4 \mu \mathrm{m} /$ h) increased after the intervention, with an associated decrease in the number of slow-/nonmigratory cells (velocity $<4 \mu \mathrm{m} / \mathrm{h})(\mathrm{Figure} 2 \mathrm{~A})$. On average per tumor, we observed a $1.75(\mathrm{SD}=0.16)$-fold increase in the percentage of migratory cells when a biopsy-like injury was performed, compared to control mice that were not biopsied (Figure 2B). We monitored tumor cell behavior for another week and found that, although the percentage of migratory tumor cells eventually decreased in both the control and the biopsied mice, the biopsied mice still exhibited a higher migratory capacity than the control mice (Figure 2C). The analysis of tumor cell proliferative behavior over time showed a 1.52 (SD $=0.26)$-fold increase in the number of mitotic events upon biopsy, relative to nonbiopsied control mice (Figure 2D).

To test whether the observed effects of biopsy on tumor cell proliferative and migratory behavior was an artifact due to CIW replacement surgery (required to perform a biopsy-like injury), we monitored tumor cell behavior in a group of mice that underwent CIW replacement without a biopsy. In this group, we did not observe any induction of migration or proliferation of tumor cells, indicating that the boost in tumor cell proliferation and migration rates were specifically triggered by biopsy-like injury (Figure $\mathbf{3 A}, \mathbf{B}$ ). 
Stable photo-convertibility of the fluorescent protein Dendra2 allows for studying tumor cell infiltration over several days. Upon exposure to ultraviolet/blue light, Dendra2 is irreversibly switched from green to red. Using this property, a square region of the tumor was illuminated and 200 Dendra2-expressing tumor cells were photo-marked before biopsy (Figure 4). One day after the biopsy, we relocalized the photo-switched region and measured the volume of tumor cells that had infiltrated into the surrounding tumor tissue. We found that the infiltration area was 1.72 $(S D=0.41)$ times larger in tumors after a biopsy-like injury compared to nonbiopsied control tumors (Figure 4). Although this approach only provides information on tumor cell bulk infiltrative behavior and not on a single cell level, it is less time-consuming than the time-lapse imaging approach and can be the method of choice for research questions focused exclusively on studying infiltrative behavior.

A
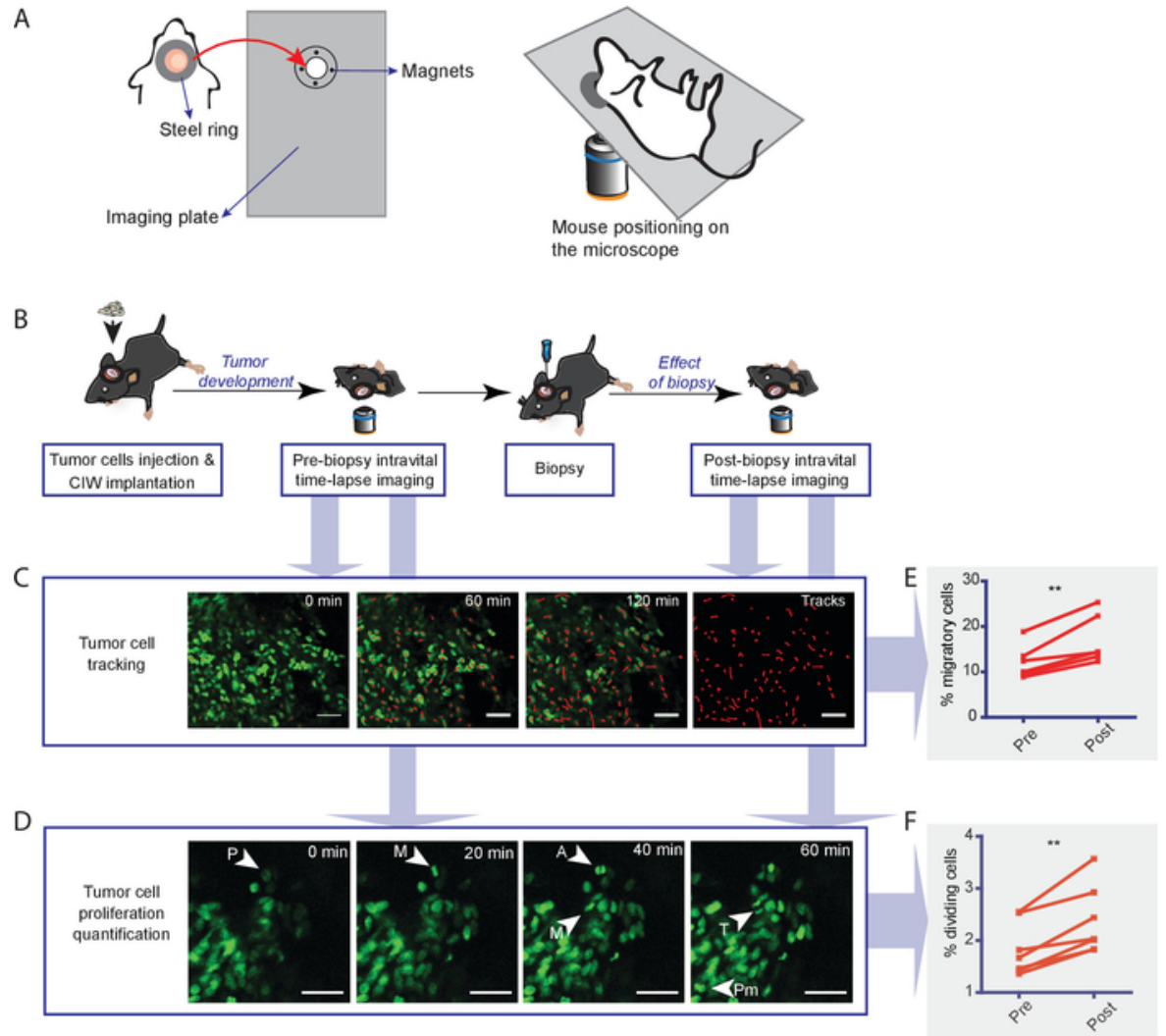

Figure 1: Experimental setup for longitudinal intravital imaging of the biopsy effect on tumor cell behavior. (A) Diagram showing the design of the ring and the magnetic holder. (B) Schematic representation of the experimental workflow. Tumor cells are injected into the brains of mice and a CIW is established. Upon tumor development, a first (prebiopsy) time-lapse imaging session is performed. The next day, the biopsy and CIW replacement are implemented. The day after imaging (postbiopsy), a second time-lapse imaging session is performed. For long-term effects, subsequent imaging sessions can be done. (C) Images show representative snapshots of a time-lapse movie where GL261 H2B-Dendra2 tumor cells were tracked. Red lines depict individual tumor cell tracks. The scale bar $=50 \mu \mathrm{m}$. (D) Representative in vivo timelapse images displaying dividing cells in GL261 H2B-Dendra2 tumors. Different stages of mitosis are indicated: prophase (P), prometaphase $(P m)$, metaphase $(M)$, anaphase $(A)$, and telophase $(T)$. The scale bar $=50 \mu \mathrm{m}$. Graphs indicate the percentage of $(E)$ migratory and $(F)$ dividing cells pre- and postbiopsy. Each dot indicates the percentage of migratory cells in all the positions measured in an individual animal. The data are shown as mean \pm S.E.M. of six mice $\left({ }^{* \star} P<0.01\right.$, paired $t$-test). This figure has been modified from Alieva et al. ${ }^{4}$. Please click here to view a larger version of this figure. 
A

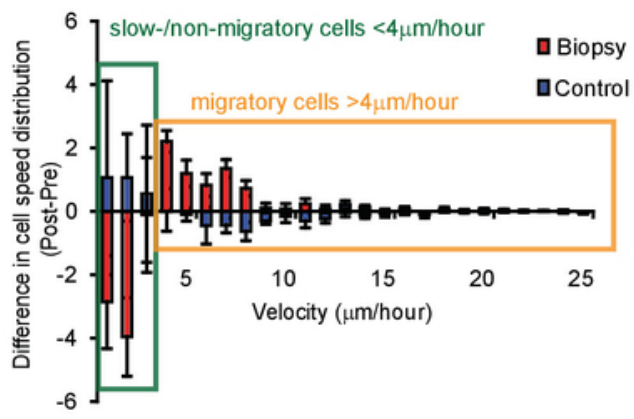

C

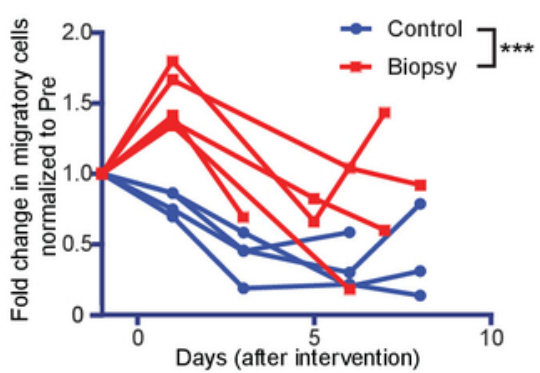

B

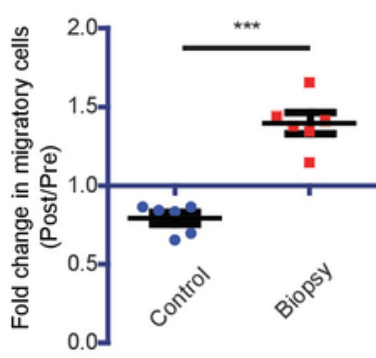

D

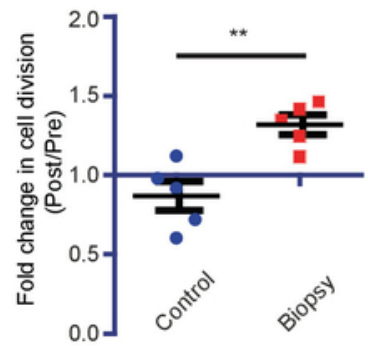

Figure 2: Representative results showing the impact of biopsy on tumor cell migration and proliferation rates. (A) Waterfall plots showing the change in cell velocity distribution relative to basal migration in individual mice. The data are shown as mean \pm S.E.M. of five mice. (B) The number of migratory cells in control (blue) and biopsied (red) animals normalized to the number of migratory cells preintervention ( $n=6$ mice, ${ }^{* * *} P<0.0001$, Student's $t$-test). (C) Tumor cell behavior was tracked over several days. Shown are the normalized (relative to preintervention) number of migratory cells in individual mice over time $\left(n>4\right.$ mice per condition, ${ }^{\star * \star} P<0.0001$, two-way ANOVA). (D) The normalized number of dividing cells in control (blue) and biopsied (red) animals. Per individual animal, the values postintervention were normalized to the values preintervention $\left(n=5\right.$ mice, ${ }^{\star \star} P<0.01$, Student's $t$-test). This figure has been modified from Alieva et al. ${ }^{4}$. Please click here to view a larger version of this figure.

A

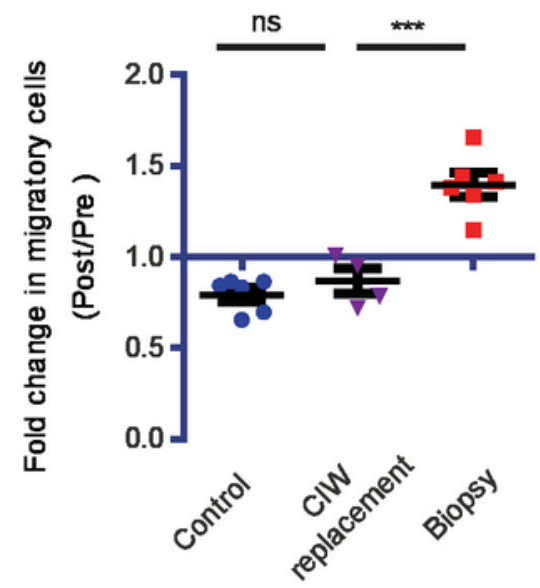

B

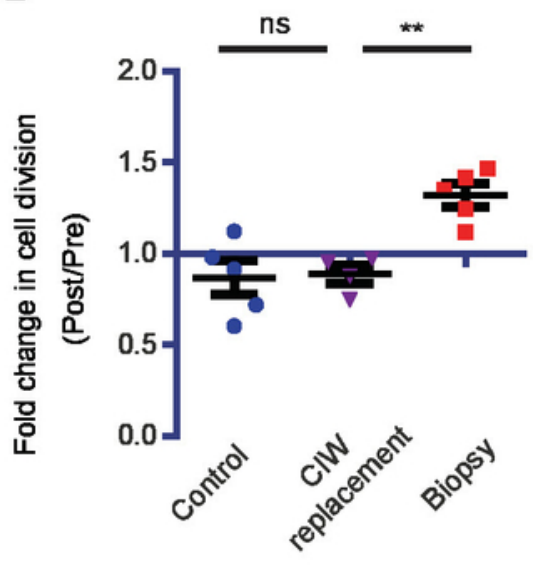

Figure 3: CIW replacement has no effect on tumor cell behavior. Longitudinal intravital imaging shows that the replacement of the CIW without biopsy has no effect on migration and proliferation rates. (A) The increase in the number of migratory cells for the indicated conditions. Every symbol represents the mean of an individual mouse, and $n \geq 4$ mice. (B) The increase in the number of proliferating cells for the indicated conditions. Every symbol represents the mean of an individual mouse ( $n \geq 4$ mice, ${ }^{\star \star} P<0.01,{ }^{* \star \star} P<0.001$, ns $=$ nonsignificant, one-way ANOVA with Newman-Keuls post hoc test). This figure has been modified from Alieva et al. ${ }^{4}$. Please click here to view a larger version of this figure. 


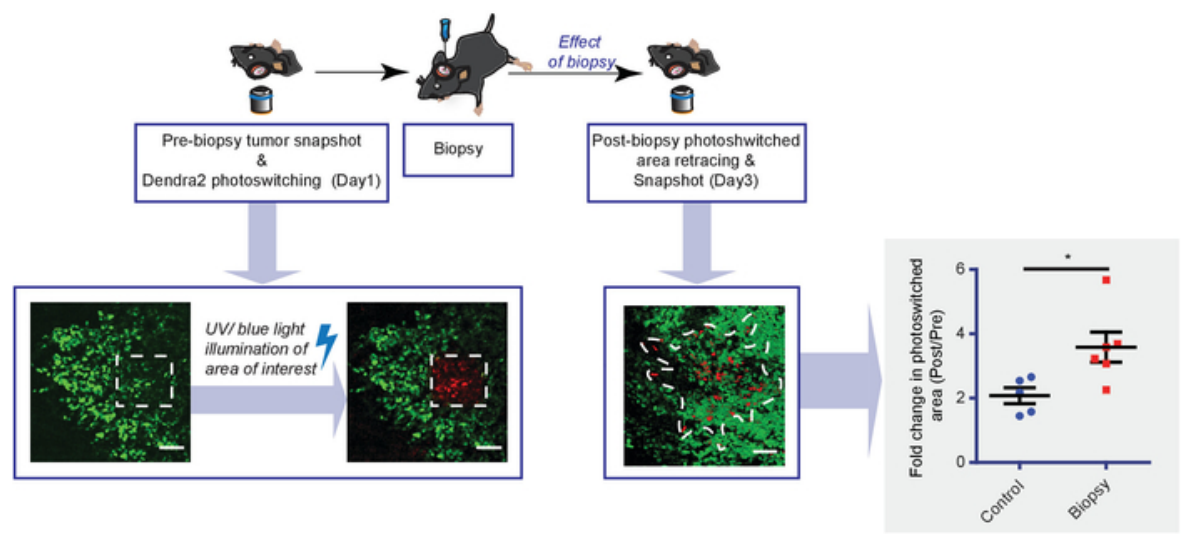

Figure 4: Diagram showing the experimental setup and representative results obtained with Dendra2 photo-switching. To monitor tumor cell infiltration upon biopsy, Dendra2-expressing tumor cells are photo-switched in a square region by UV/blue light illumination and imaged, 1 day before the biopsy. One day after the biopsy, the photo-switched region is relocalized and reimaged. Shown are representative Dendra2 images of tumor cell infiltration, corrected using channel subtraction. The white dotted line represents the infiltration area. The scale bar $=50 \mu \mathrm{m}$. The graph shows the increased photo-switched area plotted for biopsied (red) and control (blue) mice. Every dot represents the mean value of an individual mouse ( $n \geq 5$ mice, ${ }^{\star} P<0.05$, Student's $t$-test). This figure has been modified from Alieva et al. ${ }^{4}$. Please click here to view a larger version of this figure.

\section{Discussion}

Here we describe a method to study changes in tumor cell behavior at single cell level in response to invasive surgical procedures, such as a biopsy, in the brain of a living animal. The combination of longitudinal multiphoton imaging with the surgical implantation of a chronic CIW enables the quantification of tumor cell migration, invasion, and proliferation before and after biopsy in the same animal ${ }^{4}$. Compared to other approaches used for tumor multiday monitoring, such as bioluminescent imaging ${ }^{12}, \mathrm{MRI}^{10}$, or PET/CT ${ }^{11}$, this method uniquely visualizes tumors on a single cell level and, thus, provides insight in cellular behavior underlying tumor progression.

To successfully perform this method, several procedures should be mastered. The most critical steps of this protocol are CIW implantation and replacement. The technical complexity of these steps requires precision and surgical skills that can be acquired with steady training. Complications during CIW surgery, such as bleeding which may cover the brain surface, may prove challenging for subsequent imaging. A lack of sterile tools or environment, as well as the failure to completely seal the brain surface, may cause an infection on the brain surface (white liquid under the coverslip), which will make imaging problematic and strongly compromise the resulting interpretation. Another common issue of this protocol is animal movement during the time-lapse imaging. While any $x y z$ shift can be corrected after the experiment, it is recommended to correct the coordinate of each position before each time-point to prevent any loss of information. Tissue deformation is an additional problem found when imaging on an inverted microscope. Brain tissue suffers from compression when the mouse is placed in supine position. Depending on the degree of tissue deformation, tumor cell tracking may lead to an erroneous quantification of cell displacement. To prevent this, a software for rigid and elastic deformation may be used ${ }^{14}$.

While this procedure offers a broad application for studying changes in tumor behavior, certain limitations should be considered. This method allows scientists to image up to a depth of $1.6 \mathrm{~mm}$ (with the use of an optical parametric oscillator); however, this means that imaging is restricted to superficial brain cortex areas ${ }^{15}$. Thus, some brain tumors located in deep brain structures, including diffuse intrinsic pontine gliomas located in the brainstem region, cannot be studied in their original brain environment with this protocol. Another limitation of this protocol is the volume of the tumor that can be imaged. Although total tumor volume scanning is desired to obtain maximal information, often, tumor size and the speed of migratory cells can be limiting factors. For each tumor type, an optimal time-lapse for imaging has to be considered. If the time frame between images is too long, it may be difficult to track the tumor cells. The use of a resonant scanner can highly decrease scanning time, allowing the imaging of a bigger tumor ${ }^{16}$. Finally, the manual image analysis of this protocol can be very time-consuming, so instead, programs for automated 3D tracking can be used. However, the outcome of tracking should always be visually supervised since algorithms for automated cell tracking are rarely designed to recapitulate exactly the migration of the cells of interest.

Slight adaptations of the protocol described here can enable an even wider range of applications. Instead of performing biopsies, other (surgical) interventions may be implemented, such as partial tumor resection or the delivery of chemotherapy wafers. The addition of compounds through a surgically implanted microtube may be combined with this protocol to pharmacologically target specific molecules of interest. We expect that this model will be useful in studies aiming to analyze the impact of a certain intervention on tumor cell behavior. The possibility of performing repeated measures in the same animal not only provides more accurate data on changes occurring in the tumor but also greatly reduces the number of experimental animals needed per study.

\section{Disclosures}

The authors have nothing to disclose. 


\section{Acknowledgments}

The authors thank Anko de Graaff and the Hubrecht Imaging Center for their imaging support and Ellen Wehrens and Hannah Johnson for proofreading and editing the manuscript.

\section{References}

1. Heuckmann, J. M., Thomas, R. K. A new generation of cancer genome diagnostics for routine clinical use: overcoming the roadblocks to personalized cancer medicine. Annals of Oncology. 26, 1830-1837 (2015).

2. van Malenstein, $\mathrm{H}$. et al. Histology obtained by needle biopsy gives additional information on the prognosis of hepatocellular carcinoma. Hepatology Research. 42, 990-998 (2012).

3. Kretschmer, L. et al. High incidence of in-transit metastases after sentinel node biopsy in patients with melanoma (Br J Surg 2004; 91 : 1370-1371). British Journal of Surgery. 92, 253-254 (2005).

4. Alieva, M. et al. Preventing inflammation inhibits biopsy-mediated changes in tumor cell behavior. Scientific Reports. 7, 7529 (2017).

5. Hobson, J. et al. Acute inflammation induced by the biopsy of mouse mammary tumors promotes the development of metastasis. Breast Cancer Research Treatment. 139, 391-401 (2013).

6. Weil, S. et al. Tumor microtubes convey resistance to surgical lesions and chemotherapy in gliomas. Neuro-Oncology. 19, 1316-1326 (2017).

7. Parsa, A. T. et al. Prognostic significance of intracranial dissemination of glioblastoma multiforme in adults. Journal of Neurosurgery. 102, 622-628 (2005).

8. Zomer, A. et al. Intravital imaging of cancer stem cell plasticity in mammary tumors. Stem Cells. 31, $602-606$ (2013).

9. Ritsma, L. et al. Intravital microscopy through an abdominal imaging window reveals a pre-micrometastasis stage during liver metastasis. Science Translational Medicine. 4, 158ra145 (2012).

10. Schreurs, T. J. et al. Quantitative Multi-Parametric Magnetic Resonance Imaging of Tumor Response to Photodynamic Therapy. PLOS ONE. 11, e0165759 (2016).

11. Nielsen, C. H. et al. PET imaging of tumor neovascularization in a transgenic mouse model with a novel 64Cu-DOTA-knottin peptide. Cancer Research. 70, 9022-9030 (2010).

12. Alieva, M. et al. Glioblastoma therapy with cytotoxic mesenchymal stromal cells optimized by bioluminescence imaging of tumor and therapeutic cell response. PLOS ONE. 7, e35148 (2012).

13. Gligorijevic, B., Kedrin, D., Segall, J. E., Condeelis, J., van Rheenen, J. Dendra2 photoswitching through the Mammary Imaging Window. Journal of Visualized Experiment. (28), e1278 (2009).

14. Noordmans, H. J., Roode, R. d., Staring, M., \& Verdaasdonk, R. Registration and analysis of in vivo multispectral images for correction of motion and comparison in time. Vol. 6081 PWB SPIE (2006).

15. Kobat, D., Horton, N. G., Xu, C. In vivo two-photon microscopy to 1.6-mm depth in mouse cortex. Journal of Biomedical Optics. 16, 106014 (2011).

16. Kirkpatrick, N. D. et al. Video-rate resonant scanning multiphoton microscopy: An emerging technique for intravital imaging of the tumor microenvironment. IntraVital. 1 (2012). 